\title{
AN Advising System For PARKINg Using CANNY AND K-NN TECHNIQUES
}

\author{
Chyi-Ren Dow, Wei-Kang Wang, Huu-Huy Ngo, and Shiow-Fen Hwang \\ Department of Information Engineering and Computer Science Feng China \\ University, Taichung, Taiwan
}

\begin{abstract}
This study proposes a system which provides the parking characteristics and an application service platform. This system can be used to assist in selecting the parking space for drivers. The system can identify the contours of vehicles, such as cars and motorcycles by using the Canny algorithm. The data can be used to create the dataset and calculate the Parking density. Next, we use the k-nearest neighbour $(K-N N)$ algorithm to produce the parking pattern. The model makes predictions for different conditions at different time.
\end{abstract}

\section{KEYWORDS}

Parking Space, Big Data of Traffic, k-Nearest Neighbour, Canny Edge Detection.

\section{INTRODUCTION}

In modern metropolitan areas, it is difficult to park a car on the roadsides. Drivers have to spend more time looking for temporary parking space. Sometimes, they cannot find any parking space. There are many examples in real life, such as when parents need to take kids to school. The tourists need to pause their cars to buy gifts. Office workers need to go out of the office for business. For the above situations, those people need a short-term parking space to meet this dilemma.

Temporary parking or road-side parking is commonplace in the urban area. However, illegal temporary parking has different risk levels of causing accidents which depend on the time, location and road. If we can know the parking characteristics (pattern) of this area, we can park more safely and temporarily.

We want to develop a system which can provide the parking characteristics of each road to the driver to park safely. In order to solve the above problem, we expect to develop a mobile device application that will provide users with practical parking slots forecasting and real-time parking analysis based on computer vision technique.

The rest of this paper is organized as follows. Section 2 introduces the current research and technology. Section 3 discusses in detail about our system architecture. Section 4 describes the system implementation and prototype. Section 5 shows our experimental results. Finally, Section 6 summarizes the conclusions. 


\section{RELATED WORK}

In this Section, we will introduce the overview of some schemes of vehicle and parking space detection, some methods about computer vision and the traffic data analysis.

In detecting vehicles and parking spaces, Ling et al. [4] used IoT technology to detect parking spaces for vehicles and developed a notification system. Postigo et al. [8] used background subtraction method for vehicle detection and counting. Yu et al. [11] established a prediction model for parking space usage based on the historical data of reconnaissance vacancies. In the motorcycle detection, Chiu et al. [2] used the tradition method based on the gray level of the image. Duan et al. [3] used the camera for detecting the motorcycle in the real-time.

Data analysis is a process of inspecting, cleansing, transforming, and modeling data with the goal of discovering useful information, suggesting conclusions, and supporting decision-making. Data analysis has multiple facets and approaches, encompassing diverse techniques under a variety of names, in different business, science, and social science domains. Because the amount of historical traffic data is too large, we need to use algorithms such as k-nearest neighbor or random forest which can help us to rectify and find out the law. Wang et al. [10] used an outlier detection algorithm to do data preprocessing on traffic big data. Alam et al. [1] used linear regression, sequential minimal optimization (SMO) regression, and M5 base regression tree to find out the pattern from historical traffic big data. Wang et al. [9] developed the integrated platform for smart traffic big data which assist the city officials to design urban freight policies that effectively balance the needs of citizens and consumers.

Computer vision is how computer can be made for understanding from digital images or videos. For object detection and object segmentation, there are some methods such as background subtraction, optical flow, machine learning and so on. Liu et al. [5] used background subtraction method for moving object detection which got good performance. Makino et al. [6] used background subtraction method and optical flow method to detect moving object with several cameras. In recent years, due to the enhancement of computer hardware performance, addition of graphics cards, and the development of such technologies as Convolutional Neural Networks (CNN) work, the field of computer vision has drawn great attention. In terms of computer vision, Nyambal et al. [7] used CNN to detect parking spaces and predict when the parking spaces is available. CNN model can be used to combine background subtraction method. Zeng et al. [12] proposed multiscale fully convolutional network for background subtraction. 


\section{SYSTEM ARChITECTURE}

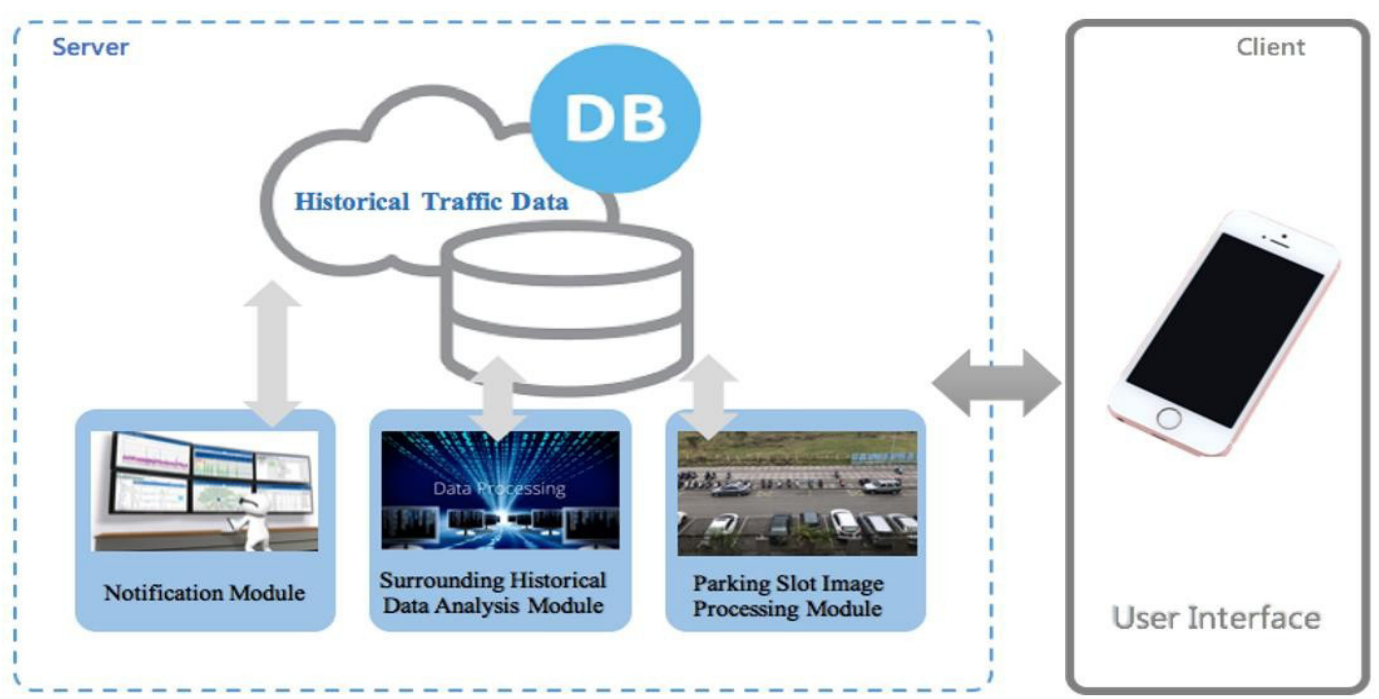

Figure 1. System Overview

This section presents the architecture of the system. As shown in Figure 1, the system consists of three subsystems, the first is the analysis and forecasting of historical traffic data system. The second is a real-time image processing system. The third is the real-time monitoring system. There are several small models constituted by the monitor image storage system, image processing server, notification service server and the mobile device applications.

The monitor video uses Ethernet to transmit the images to the server. Next, the server will recognize the image and analyze it. While the user sends a request by a mobile phone, the server will response recognition results to the user. Finally, the user gets rapid and useful information on parking space and parking slot. We used the UAV for taking parking space's picture and counting the motorcycles. The UAV can fly at most 500 meters and with a $4 \mathrm{~K}$ camera. It can take clear pictures. In the beginning, we're trying for counting the motorcycles. We separated the parking space into the parts and each part contains several lines. Each line separately contains cars and motorcycles. After counting the numbers, we restored these data into the database for linear regression to find the parking pattern. There are three different types of information needed to be stored. The first type, the empty parking space in the video and the number of motorcycles. The second type is the illegal parking information around this area, such as latitude data, longitude data, and time. The third type is the parking space information which subscribed by users.

When the camera fetches the image of parking lots. It will send to the real-time image processing module. We will use two image recognition methods for image recognition. The first method is modified from the background subtraction method which detects the difference between the two images to determine whether the object is on the screen. If there are two images in our data sets, one of the images is an empty parking space and another one has a car, we can detect the difference between two sets. We can also find how many parking spaces are available. The second method is a more accurate identification method: supervised learning. It is able to cope with a large sample dataset and train a model by itself to identify the parking spaces and motorcycles in theimage.

We provide an APP as a visualization system for users, which contains two main functions. The one is getting the information where's police towing cars which we named it "Dangerous Report Function". Another is getting real-time video in parking space and the pattern of parking which named "Parking Space Analysis Function". After data preprocessing and linear regression, we can 
get the student's parking pattern. We can show the simple line chart for users by traditional statistic method. We can also provide a prediction for users with linear regression.

\section{System Prototype}

A prototype was developed to verify the efficiency of the proposed system. Details of the prototype are presented in this section. The hardware used a Raspberry Pi 3 Module B29, which was equipped with an ARM Cortex-A53 (CPU $1.2 \mathrm{GHz}$, 64-bit, quad-core, and 1 GB RAM). A Logitech C615 webcam was used to input the video stream. An UAV records the image of parking space. We selected the East-Gate parking space in Feng Chia University, Taichung, Taiwan to implement the system. The parking spaces are a square with $165 \times 80$ square meters. The total car and motorcycle capacity is 1440 at most. We separated the parking space into many parts and lines, as shown in Figure 2.

We focus on the application for the smart mobile device which can be divided into two parts. The first part is the surrounding danger detection page which presents levels with colors. The color is calculated by the server. The green color means safe, yellow color means medium and red color means danger, as shown in Figure 3.

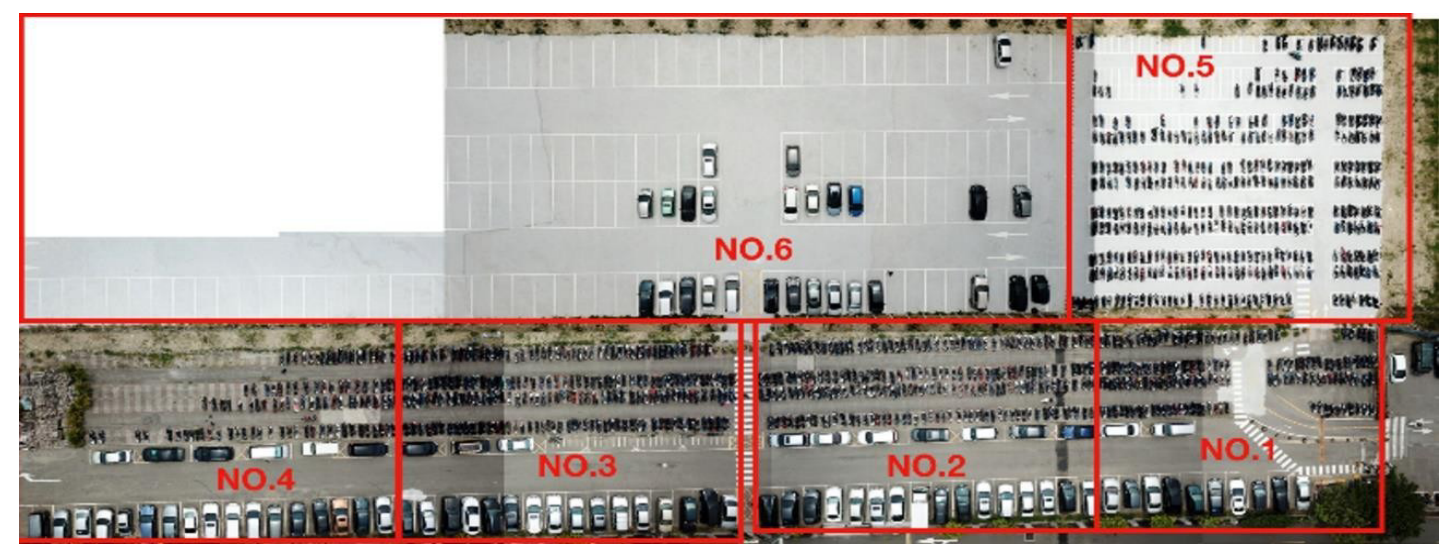

Figure 2. The parking space

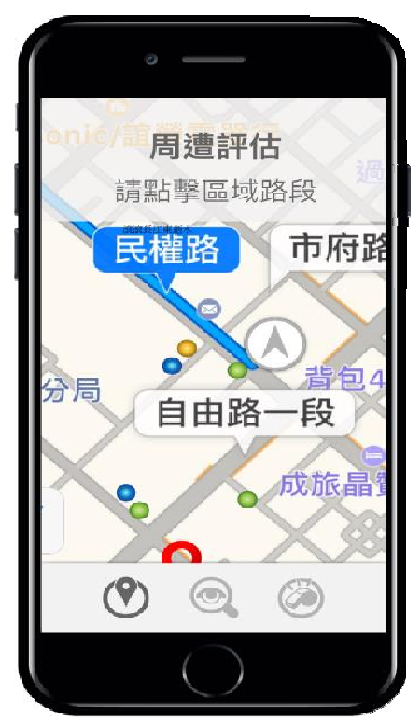

Figure 3. Surrounding Analysis Prototype 
Figure 4 shows the view which is on the mobile APP. There are three cameras to choose from the user. Users can get the information of parking pattern through watching the real-time video or the line chart. The line chart which we provide is from regression analysis and statistics. Students can get the information when they come to parking space for finding a better parking slot.

The real-time monitor system contains the dangerous decision system and the push notification service. When the user is reporting the danger, the dangerous decision system will make the decision whether to push notification to the users. The real-time image processing module which collects the image from the same camera at different times. Here are three cameras in our system providing user watching and collect images. We can convert the image to the full level of parking space's status and separate the parking space into several grids then we can count easier. To get the daily pattern of parking, the linear regression is a good function to do this purpose. We want to find the relationship in these variables which are in our databases. We choose the total number of motorcycles and daily time to do the linear regression. We can identify the area of parking space's image and the length of edges. Each image is marked with density level. We will use the $\mathrm{k}-\mathrm{NN}$ method for classification and prediction.

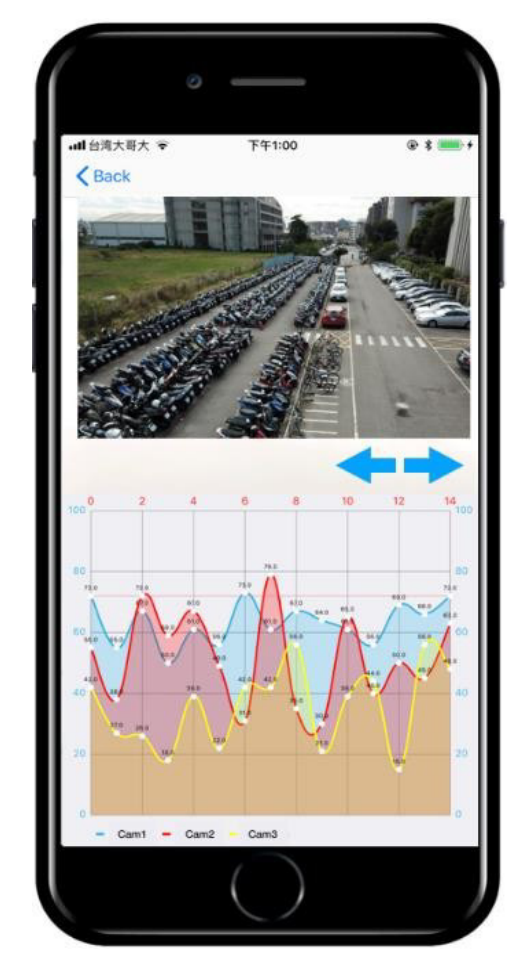

Figure 4. The APP view of Real-time monitor

\section{EXPERIMENTAL RESUlts}

After we use traditional statistics on the parking data which we collect in almost three months, we have some result as below. 


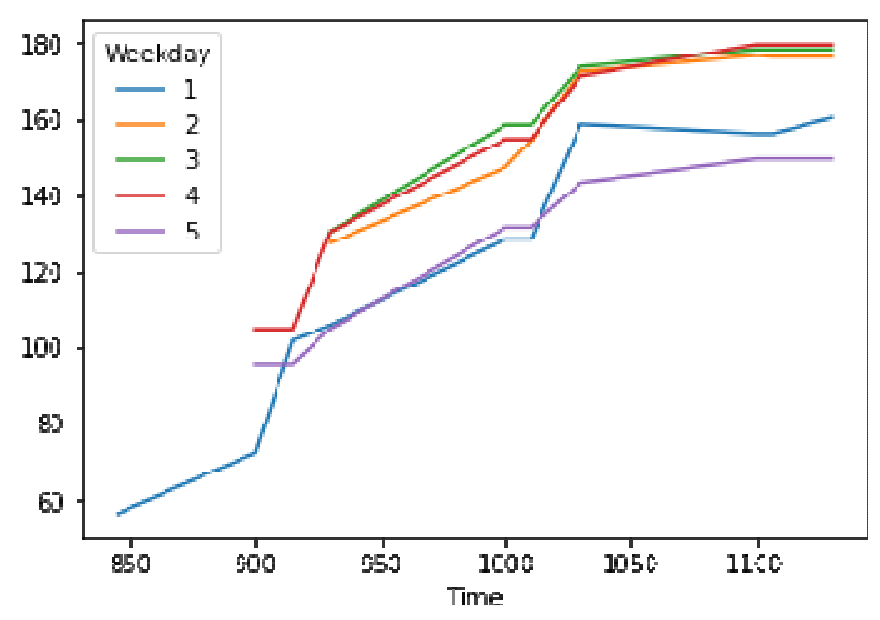

Figure 5. Time and total number of motorcycles on Weekday

As shown in Figure 5, we collect the total numbers of motorcycles from Monday to Friday to draw the line chart. We believe in the total number on Monday and Friday would be less than other days before counting. In fact, the total number on the Monday and Friday morning is obviously lower than other days until $10 \mathrm{am}$. The patterns of Tuesday, Wednesday and Thursday are very similar instead of Monday and Friday. The more interesting thing is that there is a turning point around the $10 \mathrm{am}$. That is means students have a class begin at $10 \mathrm{o}$ 'clock.

Figure 6 shows the location of parking space, time and the total number of motorcycles. The first obvious thing is that the line has a little turning down between $11 \mathrm{am}$ and 11:30 am. Next, we can see the curves of NO 1, NO 2 and NO 3 tend to be flat after 9:30 am. We guess that there are few of the parking lots in NO 1, NO 2 and NO 3 so that student only can choose NO 4 parking space.

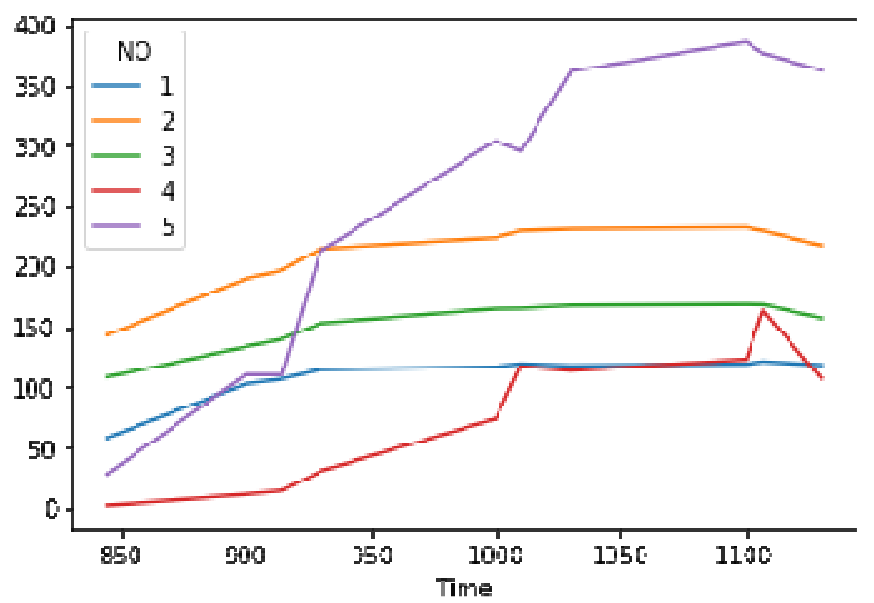

Figure 6 . The relationship between Time and total number of motorcycles

\section{Conclusions}

This paper uses Open CV, K-NN algorithm and Canny edge detection algorithm to design and implement a temporary parking guidance system. Our system consists of the historical analysis and forecasting of traffic data, the real-time image processing system and the real-time monitoring system. On the other hand, we establish the mobile app which helps drivers to park without worrying. The App can also show the risk level of user's location accurately to help user making parking decision. If the risk level is too low for short-term parking, the map view is able to show 
more information of nearby road. In addition, the monitor function is the best part of this APP. When the risk level increases or the policeman gives a ticket and impounds cars, it will notify the nearby drivers to move their car out of the risky region.

\section{REFERENCES}

[1] I. Alam, M. F. Ahmed, M. Alam, J. Ulisses, D. M. Farid, S. Shatabda and R. J. F. Rossetti, "Pattern Mining from Historical Traffic Big Data," in Proceedings of IEEE Region 10 Symposium (TENSYMP), Cochin, India, pp. 1-5, Jul. 2017.

[2] C. Chiu, M. Ku and H. Chen, "Motorcycle Detection and Tracking System with Occlusion Segmentation," in Proceedings of Eighth International Workshop on Image Analysis for Multimedia Interactive Services (WIAMIS '07), Santorini, Greece, pp. 32-32, Jun. 2007.

[3] B. Duan, W. Liu, P. Fu, C. Yang, X. Wen and H. Yuan, "Real-Time On-road Vehicle and Motorcycle Detection Using a Single Camera," in Proceedings of IEEE International Conference on Industrial Technology, Gippsland, VIC, Australia, pp. 1-6, Feb. 2009.

[4] X. Ling, J. Sheng, O. Baiocchi, X. Liu and M. E. Tolentino, "Identifying Parking Spaces \& Detecting Occupancy Using Vision-based IoT Devices," in Proceedings of Global Internet of Things Summit (GIoTS), Geneva, Switzerland, pp. 1-6, Jun. 2017.

[5] W. Liu, H. Yu, H. Yuan, H. Zhao and X. Xu, "Effective Background Modelling and Subtraction Approach for Moving Object Detection,” IET Computer Vision, vol. 9, no. 1, pp. 13-24, Feb. 2015.

[6] K. Makino, T. Shibata, S. Yachida, T. Ogawa and K. Takahashi, "Moving-Object Detection Method for Moving Cameras by Merging Background Subtraction and Optical Flow Methods," in Proceedings of IEEE Global Conference on Signal and Information Processing (GlobalSIP), Montreal, QC, Canada, pp. 383-387, Nov. 2017.

[7] J. Nyambal and R. Klein, "Automated Parking Space Detection Using Convolutional Neural Networks," in Proceedings of Pattern Recognition Association of South Africa and Robotics and Mechatronics (PRASA-RobMech), Bloemfontein, South Africa, pp. 1-6, Nov. 2017.

[8] C. G. del Postigo, J. Torres and J. M. Menéndez, "Vacant Parking Area Estimation Through Background Subtraction and Transience Map Analysis," IET Intelligent Transport Systems, vol. 9, no. 9, pp. 835-841, Nov. 2015.

[9] X. Wang and Z. Li, "Integrated Platform for Smart Traffic Big Data," in Proceedings of International Conference on Logistics, Informatics and Service Sciences (LISS), Sydney, NSW, Australia, pp. 1-6, Jul. 2016.

[10] Z. Wang, X. Huang, Y. Song and J. Xiao, "An Outlier Detection Algorithm Based on The Degree of Sharpness and Its Applications on Traffic Big Data Preprocessing," in Proceedings of IEEE 2nd International Conference on Big Data Analysis (ICBDA)(, Beijing, China, pp. 478-482, Mar. 2017.

[11] F. Yu, J. Guo, X. Zhu and G. Shi, "Real Time Prediction of Unoccupied Parking Space Using Time Series Model," in Proceedings of International Conference on Transportation Information and Safety (ICTIS), Wuhan, China, pp. 370-374, Jun. 2015.

[12] D. Zeng and M. Zhu, "Background Subtraction Using Multiscale Fully Convolutional Network," IEEE Access, vol. 6, pp. 16010-16021, 2018. 


\section{AUTHORS}

Chyi-Ren Dow received the B.S. and M.S. degrees in Information Engineering from National Chiao Tung University, Taiwan, in 1984 and 1988, respectively, and the M.S. and Ph.D. degrees in Computer Science from the University of Pittsburgh, USA, in 1992 and 1994, respectively. Currently, he is a Professor in the Department of Information Engineering, Feng Chia University, Taiwan. His research interests include mobile computing, ad hoc wireless networks, agent techniques, computer vision and deep learning.

Wei-Kang Wang received the B.S. and M.S. degree from the Department of Information Engineering and Computer Science, Feng Chia University, Taiwan, in 2014 and 2018, respectively. His research interests include IoT, big data, data mining and machine learning.

Huu-Huy Ngo received his B.S. and M.S. degrees from Thai Nguyen University of Information and Communication Technology, Vietnam, in 2010 and 2012, respectively. Currently, he is pursuing the Ph.D. degree with the Department of Information Engineering and Computer Science, Feng Chia University, Taiwan. His research interests include machine learning, computer vision and image recognition.

Shiow-Fen Hwang received her B.S., M.S. and Ph.D. degrees in Applied Mathematics from National Chiao Tung University, Taiwan, in 1985, 1987 and 1991, respectively. Currently, she is a Professor in the Department of Information Engineering, Feng Chia University, Taiwan. Her research interests include interconnection networks, mobile computing and computer algorithms.
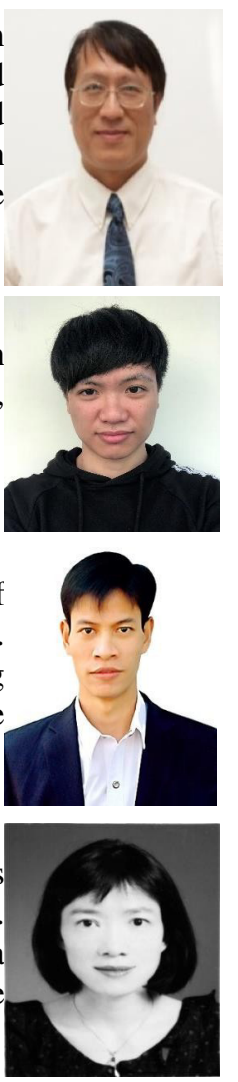